\title{
HIGH IMPEDANCE SURFACES FOR FLEXIBLE AND CONFORMAL WIRELESS SYSTEMS
}

\author{
${ }^{1}$ Haider R. Khaleel, ${ }^{2}$ Hussain M. Al-Rizzo, ${ }^{2}$ Ayman Issac and ${ }^{2}$ Said Abushamleh \\ ${ }^{1}$ Department of Engineering Science, Sonoma State University, Rohnert Park, California, USA \\ ${ }^{2}$ Department of Systems Engineering, University of Arkansas at Little Rock, Little Rock, Arkansas, USA
}

Received 2012-12-10; Revised 2014-06-10; Accepted 2014-06-25

\begin{abstract}
Recent years have witnessed a great deal of interest from both scientific and academic communities in the field of flexible electronic systems. Most flexible electronic systems require the integration of flexible antennas operating in specific frequency bands to provide wireless connectivity which is highly demanded by today's network oriented society. On the other hand, High Impedance Surfaces have become very popular in the design of contemporary antenna and micro-wave devices due to their wide range of applications derived from their unique electromagnetic properties which significantly enhance the performance of antennas and RF systems. Accordingly, the integration of HIS structures within flexible wireless systems is very beneficial in this growing field of research. In this paper, a systematic review of flexible HIS structures reported in the literature is conducted, which provides the reader with a thorough description and a complete list of state of the art designs intended for flexible wireless systems.
\end{abstract}

Keywords: High Impedance Surface (HIS), Antennas, Flexible Electronics, Wireless Systems

\section{INTRODUCTION}

Today's industrial and academic research is focusing intensive activities aimed at developing flexible and wearable wireless systems due to their applicability in a wide spectrum of applications such as personal communication, medicine, firefighting, entertainment, aeronautics, military and Radio Frequency Identification (RFID) tags (Cai et al., 2007; Hertleer et al., 2009). Their light weight, low fabrication cost, ease of manufacturing and the availability of inexpensive flexible films/substrates (i.e., papers, textiles and plastics) make flexible electronics an appealing candidate for the next generation of hand-held electronics (Nathan and Chalamala, 2005). Moreover, recent developments in miniaturized and flexible energy storage components paved the road for the commercialization of such systems (Huang et al., 2011).

Modern flexible electronics and gadgets such as flexible mobile phones, displays, electronic books and rollable keyboards are often equipped with an antenna to provide wireless connectivity. Needless to say, the efficiency of these systems primarily depends on the characteristics of the integrated antenna.

The nature of flexible wireless technologies requires the integration of flexible, low profile, light weight and compact antennas. At the same time, these antennas should be mechanically robust, efficient with sufficient bandwidth and desirable radiation characteristics.

On the other hand, the same decade witnessed the revolution of Metamaterials (MTMs), artificially engineered structures which exhibit unique electromagnetic properties that do not exist in nature (Yao et al., 2005). These structures can be engineered to have desired features within a desired frequency range. The integration of these artificial structures with antennas and microwave circuits has shown to be extremely beneficial in several applications (Yang and Rahmat-Samii, 2009).

The most promising types of MTMs that are expected to be industrialized in the field of wireless systems are Corresponding Author: Haider R. Khaleel, Department of Engineering Science, Sonoma State University, Rohnert Park, California, USA 
the $\mu$-Negative (MNG) MTM and High Impedance Surfaces (HIS) (Sievenpiper et al., 1999).

HISs are successfully applied in many antenna systems for performance enhancement utilizing their unique features. Their advantages include surface wave reduction which is considered as a serious drawback in microstrip antennas as it reduces antenna's gain, efficiency, limit their bandwidth and increase cross polarization levels. In phase reflection and mutual coupling reduction among radiating elements in antenna arrays in general and MIMO systems in particular are very promising applications of these artificial structures. However, the application of such structures in flexible and wearable technologies is limited by their relatively high profile and their size and bandwidth's dependence on the substrate's relative permittivity and thickness (Park et al., 2001). This has triggered the need for flexible, conformal and compact HISs to be integrated within the targeted technology. In response to such requirements, a plethora of designs and techniques have been reported in the literature which will be reviewed in this article.

In section 2, a theoretical background on HISs is provided. In section 3, we discuss the design, methodology and applications of the significant flexible HIS designs reported in the literature. The flexibility analysis involved with characterizing the performance of HISs under bending and flexing conditions is discussed in section 4. Finally, conclusions are given in section 5 .

\section{HIGH IMPEDANCE SURFACES: BACKGROUND}

HIS was first proposed by (Sievenpiper et al., 1999) the structure of which was based on square metallic plates printed on a grounded dielectric substrate and connected to the ground plane through metallic posts (vias). Later on, numerous types of HIS geometries have been extensively studied (Kim and Yeo, 2008; Costa et al., 2009). In general, HIS's are classified into two types based on their characteristic features: Artificial Magnetic Conductor (AMC), which mimics a Perfect Magnetic Conductor (PMC) in a specific frequency range and Electromagnetic Band Gap (EBG). It is known that a Perfect Electric Conductor (PEC) has a reflection phase of $180^{\circ}$ for a normally incident plane wave, while a Perfect Magnetic Conductor (PMC), which does not exist in nature, has a reflection phase of $0^{\circ}$. Image theorem states that a PEC ground plane causes the antenna's current and its image to cancel each other, in other words shorting the antenna. This is responsible for dropping the real part of the antenna impedance towards zero ohms, while the imaginary impedance approaches infinity. Thus, a significant amount of the electromagnetic energy is trapped between the antenna and the ground plane; hence the antenna can no longer radiate efficiently (Kim and Yeo, 2008). This is the opposite scenario if an AMC is placed instead of PEC due to its reflection of electromagnetic wave with zero phase shift.

HIS structures can be artificially engineered to have in phase reflection coefficient properties in a specified frequency range. They are typically realized based on periodic metallization patterns which are often called Frequency Selective Surfaces (FSS) printed on a grounded dielectric material (Qian et al., 1997).

The surface impedance of the HIS is defined as the ratio of the tangential component of the electric field $E$ to the tangential component of the magnetic field $H$ at the surface Equation 1:

$$
Z_{s}=\frac{\left|E_{t}\right|}{\left|H_{t}\right|}
$$

where, $E_{t}$ and $H_{t}$ are the electric and magnetic fields components tangential to the surface.

The FSS grid along with the grounded dielectric slab can be represented by a capacitance in parallel with an inductance (Costa et al., 2009), i.e., a resonant LC circuit with a resonant frequency given by Equation 2:

$$
f=\frac{1}{2 \Pi \sqrt{L C}}
$$

where, $L$ and $C$ are the equivalent inductance and capacitance associated with the dielectric slab and the FSS grid. The circuit analogy for the HIS is depicted in Fig. 1.

This resonant frequency is determined by the dimensions and geometry of the structure; i.e., changing the dimensions and geometry leads to a change in the values of $L$ and $C$ and therefore the resonant frequency can be modified accordingly. For example, the period of a square patch based HIS is related to its resonant frequency by Equation 3:

$p=\frac{\lambda}{2}=\frac{c}{2 f \sqrt{\varepsilon}}$ 


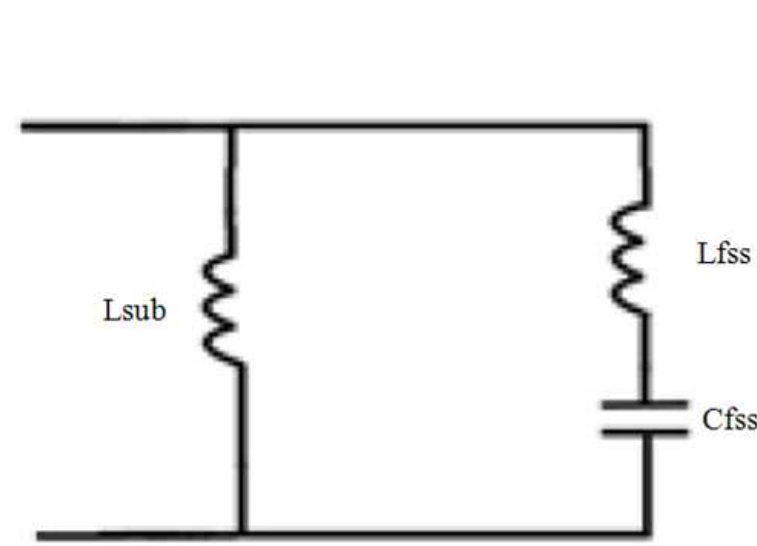

(a)

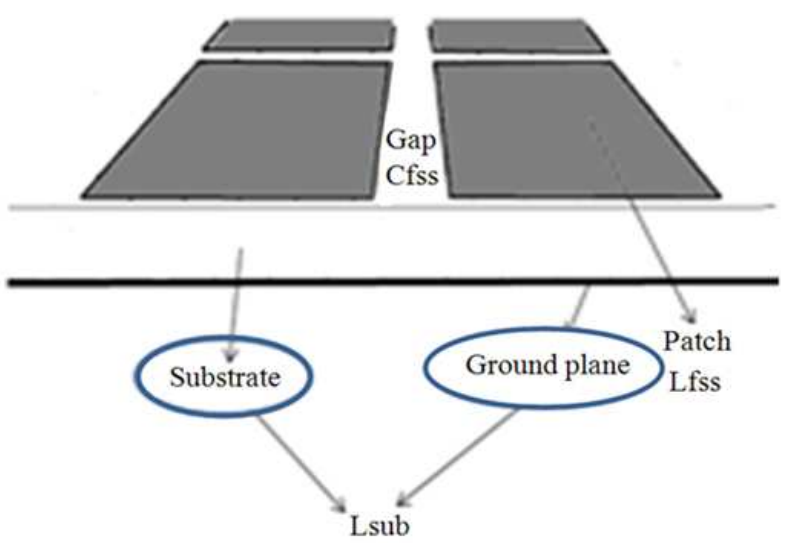

(b)

Fig.1. (a) Circuit representation for the HIS structure (b) Conventional Square-patch based HIS structure

According to the same parallel $L C$ model, the surface impedance and reflection coefficient for the HIS structure can be calculated by Equation 4 and 5:

$$
Z_{s}=\frac{j \omega L}{1-(\omega / \omega o)^{2}}
$$

where, $\omega_{0}$ is:

$\omega_{o}=\frac{1}{2 \Pi \sqrt{L C}}$

And the reflection coefficient is Equation 6:

$$
\Gamma=\frac{Z_{s}-Z_{o}}{Z_{s}+Z_{o}}
$$

where, $Z_{0}$ is the free space impedance. As can be determined from (6), the surface impedance $Z_{s}$ of a PEC surface, which has a zero tangential component of electric field is zero. Hence, the PEC reflection coefficient is equal to -1 . While for a PMC surface, the tangential component of the magnetic field is zero which yields infinite surface impedance. Thus, the plane wave reflection coefficient for the PMC is +1 .

It is worth mentioning here that the operational HIS bandwidth is between $-90^{\circ}$ and $+90^{\circ}$ as defined by (Sievenpiper et al., 1999).

On the other hand, when dealing with surface waves suppression, mushroom type EBG structure demonstrates a better performance compared to vialess structures. However, grounding vias complicate the fabrication process and it would be impractical to consider it for flexible and conformal applications.

In general, EBG's resonant frequency does not normally coincide with the AMC's. This can deteriorate the benefits of HIS in specific applications. However, the $\mathrm{AMC}$ and $\mathrm{EBG}$ features are not required to coexist in the considered application.

\section{LITERATURE REVIEW}

Bai et al. (2009) investigated the performance of a textile Coplanar Waveguide fed (CPW) antenna under bending and crumpling conditions. A HIS is used to improve the wearable antenna performance. Both input impedance and radiation pattern were investigated based on numerical and experimental methods.

Zhu and Langley (2009), investigated the performance of a flexible dual band $(2.45,5 \mathrm{GHz})$ textile antenna based on the conventional square patch based HIS in terms of Specific Absorption Rate (SAR). A significant reduction in SAR achieved when the HIS structure is included; however, the size of the design is relatively large $(120 \times 120 \mathrm{~mm})$. Moreover, textile based antennas are prone to discontinuities in substrate materials in addition to the textile nature of fluid absorption. A flexible, compact antenna system intended for telemedicine applications was proposed by (Raad et al., 2012). The design is based on an $\mathrm{M}$ shaped printed monopole antenna operating in the Industrial, Scientific and Medical (ISM) $2.45 \mathrm{GHz}$ band integrated with a miniaturized slotted Jerusalem Cross (JC) HIS ground plane. The HIS ground plane is utilized to isolate the user's body from undesired electromagnetic radiation in addition to minimizing the antenna's impedance mismatch caused by the proximity to human tissues. 
SAR is assessed using a Human body model (HUGO) to verify the feasibility of the proposed design. The antenna expresses $18 \%$ impedance bandwidth; moreover, the integration of the HIS ground plane increases the front to back ratio by $8 \mathrm{~dB}$, provides $3.7 \mathrm{~dB}$ increase in gain, in addition to $64 \%$ reduction in SAR (Shahid, 2009) presented a modified wearable form of HIS defined as non uniform HIS and integrated it with an antenna for improved performance under low profile limitation. The HIS was also utilized to reduce the size of a normal patch antenna and improve its gain and impedance bandwidth. A low profile antenna based on the properties of a non-uniform HIS was presented by the same author in 2010. The proposed design is able to minimize electromagnetic interaction with the human tissues which in turn reduces SAR and degradation in radiation efficiency. Finally, A flexible uniplanar HIS design manufactured using laser micromachining was presented by (De Cos and Las-Heras, 2012). It is characterized under flat and bent conditions by measuring its reflection coefficient phase in an anechoic chamber. The designed prototype shows broad HIS operation bandwidth (around $7 \%$ ) and polarization angle independency.

\section{FLEXIBILITY ANALYSIS}

As stated previously, flexible, wearable and conformal antenna systems are becoming extremely popular nowadays. One of the main challenges facing such systems is the uncertainty of maintaining performance parameters of the wearable/conformal antenna system which are based on their flat profile during operation especially for elements made of flexible materials. Therefore, it is necessary to evaluate the performance of antennas and any type of integrated structures under bending and flexing conditions. It is worth mentioning that most previous research was only focused on investigating the bending effects on wearable/conformal antennas based on conventional PEC ground planes both numerically and experimentally (Farahani et al., 2010). Palikaras et al. (2011), proposed a conformal HIS is utilized to reduce the diameter of a cylindrical antenna, However, only the effect of curvature on the far field radiation pattern was investigated. Bai et al. (2009) investigated the performance of a dual band textile antenna integrated with an HIS under bending and crumpling conditions in terms of input impedance and radiation patterns. However, the reported research focused mainly on the effect of curvature on the antenna characteristics only. De Cos et al. (2011) presented and characterized a flexible uniplanar AMC based on reflection coefficient phase under flat and creeping conditions. Liu et al. (2008) proposed a trial and error method to evaluate the effect of curvature on the reflection phase characteristics of an AMC structure. By observing the changes of the corresponding frequency band of return loss of different length dipoles antennas versus inphase reflection, the effect of curvature on the reflection phase is determined. Raad et al. (2012) proposed a systematic approach to characterize the performance of a flexible HIS when subjected to different extents of bending is proposed. The performance of an array of squarepatch based HIS under various bending extents was investigated as a benchmark. According to the reflection phase analysis, a shift to a higher resonant frequency is observed in addition to bandwidth degradation when the degree of bending is increased. It is worth mentioning that the reported study could be applied to different HIS geometries depending on the targeted application.

\section{CONCLUSION}

The integration of High Impedance Surfaces (HISs) within modern wireless systems is becoming increasingly popular nowadays due to their beneficial properties. Consistently, flexible electronics are drawing much attention and are on a fast track to commercialization. This triggered the need for an article to systematically review the status of the flexible and conformal HIS research.

A theoretical background was provided in the first section, followed by an extensive literature review describing design and applications of flexible HISs. Finally, flexibility analyses which are needed to characterize the performance of such structures under operational bending and flexing effects are reviewed. It was concluded that this type of study is vital when designing HIS structures for flexible and conformal applications due to their consequent parameters change.

\section{REFERENCES}

Bai, L., A.L. Goldman and J.R. Carlson, 2009. Positive and negative regulation of odor receptor gene choice in Drosophila by acj6. J. Neurosci., 29: 1294012947. DOI: 10.1523/JNEUROSCI.3525-09.2009

Cai, X.H., B. An, X.W. Lai, Y.P. Wu and F.S. Wu et al., 2007. Reliability evaluation on flexible RFID tag inlay packaged by anisotropic conductive adhesive. Proceedings of the 8th International Conference on Electronic Packaging Technology, Aug. 14-17, IEEE Xplore Press, Shanghai, pp: 1-4. DOI: 10.1109/ICEPT.2007.4441524 
Costa, F., S. Genovesi and A. Monorchio, 2009. On the bandwidth of high-impedance frequency selective surfaces. Proceedings of the IEEE Antennas and Wireless Propagation Letters, Dec. 11-31, IEEE Xplore Press, pp: 1341-1344. DOI: 10.1109/LAWP.2009.2038346

De Cos, M.E. and F. Las-Heras, 2012. Novel uniplanar flexible artificial magnetic conductor. Int. J. Antennas Propagat., 109: 1031-1035. DOI: 10.1007/s00339-012-7373-9

De Cos, M.E., Y. Alvarez, R. Hadarig and F. Las-Heras, 2011. Flexible uniplanar artificial magnetic conductor. Proceedings of the 5th European Conference on Antennas and Propagation, April 1115, IEEE Xplore Press, Rome, pp: 1218-1221.

Farahani, H.S., M. Veysi, M. Kamyab and A. Tadjalli, 2010. Mutual Coupling Reduction in Patch Antenna Arrays Using a UC-EBG Superstrate. IEEE Antennas Wireless Propagat. Lett., 9: 57-59. DOI: 10.1109/LAWP.2010.2042565

Hertleer, C., H. Rogier, L. Vallozzi and L. Van Langenhove, 2009. A textile antenna for off-body communication integrated into protective clothing for firefighters. IEEE Trans. Antennas Propagat., 57: 919-925. DOI: 10.1109/TAP.2009.2014574

Huang, Y., J. Chen, Z. Yin and Y. Xiong, 2011. Roll-toroll processing of flexible heterogeneous electronics with low interfacial residual stress. IEEE Trans. Components Packag. Manufact. Technol., 1: 13681377. DOI: 10.1109/TCPMT.2011.2157692

Kim, D. and J. Yeo, 2008. Low-profile RFID tag antenna using compact AMC substrate for metallic objects. IEEE Antennas Wireless Propagat. Lett., 7: 718720. DOI: 10.1109/LAWP.2008.2000813

Liu, T., X.Y. Cao, J. Ma and W. Wang, 2008. Effect of curvature on reflection phase characteristics of electromagnetic band-gap structures. Proceedings of the Global Symposium on Millimeter Waves, Apr. 21-24, IEEE Xplore Press, Nanjing, pp: 260-263. DOI: 10.1109/GSMM.2008.4534617

Nathan, A. and B.R. Chalamala, 2005. Special Issue on Flexible Electronics Technology, Part 1: Systems and Applications. IEEE Proc., 93: 1235-1238. DOI: 10.1109/JPROC.2005.851525

Palikaras, G.K., A.P. Feresidis and C.G. Parini, 2011. Advances in conformal metamaterial antennas using High Impedance (HIS) and Electromagnetic Bandgap (EBG) surfaces. Proceedings of the 5th European Conference on Antennas and Propagation, Apr. 11-15, IEEE Xplore Press, Rome, pp: 34663469. DOI: 978-1-4577-0250-1
Park, J.Y., C.C. Chang, Y. Qian and T. Itoh, 2001. An improved low-profile cavity-backed slot antenna loaded with 2D UC-PBG reflector. Proceedings of the IEEE Antennas and Propagation Society Symposium, Jul. 8-13, IEEE Xplore Press, Boston, MA, USA, pp: 194-197. DOI: 10.1109/APS.2001.959431

Qian, Q., M. Wu, H. Cao, Y. Guo and S. Fang et al., 1997. The effect of antisense human Fas RNA on activation induced apoptosis of $\mathrm{T}$ cell. Zhonghua, 18: 619-622. PMID: 15625758.

Raad, H.R., A.I. Abbosh, H.M. Al-Rizzo and D.G. Rucker, 2012. Flexible and compact AMC based antenna for telemedicine applications. IEEE Trans. Antennas Propagat., 61: 524-531. DOI: 10.1109/TAP.2012.2223449

Shahid, B., 2009. Design and synthesis of non uniform high impedance surface based wearable antennas. Phd thesis, Loughborough University Institutional Repository.

Sievenpiper, D., L.J. Zhang, R.F.J. Broas, N.G. Alexopolous and E. Yablonovitch et al., 1999. High-impedance electromagnetic surfaces with a forbidden frequency band. IEEE Trans. Microw. Theory Tech., 47: 2059-2074. DOI: 10.1109/22.798001

Yang, F. and Y. Rahmat-Samii, 2009. Electromagnetic Band Gap Structures in Antenna Engineering. 1st Edn., Illustrated, Cambridge, Cambridge University Press, ISBN-10: 052188991X, pp: 266.

Yao, H.Y., W. Xu, L.W. Li, Q. Wu and T.S. Yeo et al., 2005. Propagation property analysis of metamaterial constructed by conductive SRRs and wires using the MGS-based algorithm. IEEE Trans. Microwave Theory Techniques, 53: 14691476. DOI: 10.1109/TMTT.2005.845210

Zhu, S. and R. Langley, 2009. Dual-band wearable textile antenna on an EBG substrate. IEEE Trans. Antennas Propagat., 57: 926-935. DOI: 10.1109/TAP.2009.2014527 